\title{
Viscoelasticity of Potassium Neutralized Telechelic Poly(ethylene-butylene) Ionomer in Non-polar Solvent. II
}

\author{
Syed Atiqur RAHMAn and Norio Nemoto ${ }^{\dagger}$ \\ Department of Molecular and Material Sciences, IGSES, Kyushu University, Hakozaki, Fukuoka 812-8581, Japan
}

(Received April 15, 2004; Accepted July 6, 2004; Published October 15, 2004)

\begin{abstract}
Dynamic viscoelastic measurements are made on six potassium neutralized telechelic poly(ethylene butylene) ionomer (CTPEB-K) solutions in anhydrous decalin with $C$ from 7 to $70 \mathrm{wt} \%$ as well as the bulk sample for establishing viscoelastic properties of this system along with earlier data over the whole concentration range. Differences in the formation process of viscoelastic networks and their structures between the two ionomeric systems of CTPEB-K and -Mg are extensively discussed using concentration dependencies of viscosity and the number density of the elastically effective chains and also the temperature dependence of the shift factor obtained from the time-temperature reduction scheme. [DOI 10.1295/polymj.36.817]

KEY WORDS Dynamic Viscoelasticity / CTPEB-K / Network Relaxation / Ion Clusters /
\end{abstract}

Telechelic ionomers containing ionic groups only at both chain ends are useful for studying details of association processes induced by metallic ions as a model system and many fruitful results have been obtained. ${ }^{1-17}$ It seems well established that telechelic ionomers are aggregated even at very dilute concentration in anhydrous solvent, grow in aggregate size with increasing polymer concentration $C$ and then form weak viscoelastic networks at certain $C^{*}$ depending on a kind of metallic ions used followed by development of stronger networks with ion clusters as crosslinking points up to the bulk. Here, adjectives "weak" and "strong" are only conventionally used to differentiate two networks with that there are large differences in magnitudes of viscosity and plateau modulus characteristic of the viscoelastic network.

In a previous report, ${ }^{18}$ we studied in detail viscosity and dynamic viscoelastic behaviors of magnesium neutralized telechelic poly(ethylene butylene) ionomers (CTPEB-Mg) in a nonpolar solvent of decalin from very dilute solution up to the bulk. We found that CTPEB-Mg chains are associated as ring polymers in very dilute decalin solution forming a $-\mathrm{COO}^{-} \mathrm{Mg}^{2+-} \mathrm{OOC}-$ ionic bond (MDC bond) due to the divalent nature of $\mathrm{Mg}^{2+}$ cation, i.e., due to the restriction that the electrically neutral condition must be satisfied. Using the dilute polymer solution theory, ${ }^{19,20}$ we found that about five CTPEB-Mg chains are associated to each other to form one ring, and with further increase in $C$ these rings are linearly associated to each other so as to form huge polymeric clusters of various sizes. A weak viscoelastic network is formed near the overlapping concentration $C^{*}$ of the system estimated from the intrinsic viscosity value. The network becomes stronger forming ion clusters in such a way that ion clusters are mainly made of two MDC bonds up to $C=30 \mathrm{wt} \%$ and then larger and thermodynamically more stable ion clusters are formed above $C=50 \mathrm{wt} \%$.

When monovalent cation $\mathrm{K}^{+}$is used for stoichiometric neutralization of CTPEB, an ion pair of $\mathrm{COO}^{-} \mathrm{K}^{+}$is allowed to be present as a strong dipole in decalin at each chain end, since the electrically neutral condition is satisfied. However, pairwise association of two $\mathrm{COO}^{-} \mathrm{K}^{+}$ion pairs or formation of ion clusters with more than two $\mathrm{COO}^{-} \mathrm{K}^{+}$ion pairs is also possible. The simplest associating structure may be a linear type of association formed by a pairwise associate of $-\mathrm{COO}^{-} \mathrm{K}^{+}$residues at respective chain ends. It is likely that associating structures of CTPEB-K chains at very dilute solution as well as at higher concentrations and in the bulk are different from those of the CTPEB-Mg ionomer. In fact, we measured viscosity and viscoelasticity of the CTPEB-K system from dilute solution up to $C=30 \mathrm{wt} \%$ and showed that CTPEB-K chains are very slightly associated with an association number $a=1.36$ at the infinite dilution limit. ${ }^{21}$ With increasing concentration, CTPEB-K chains are almost linearly associated, resulting in formation of a viscoelastic network near its overlapping concentration lower than $C^{*}$ of the CTPEB-Mg system. We found that the viscoelastic networks are formed mainly via pairwise association of two $\mathrm{COO}^{-} \mathrm{K}^{+}$groups, and larger clusters made of more than two $-\mathrm{COO}^{-} \mathrm{K}^{+}$ionic groups that act as crosslinking points in the network are present smaller in number, though it increases with increasing $C$ up to the highest concentration $C=30 \mathrm{wt} \%$ studied. We also found the presence of a slow relaxation mode, which is ascribed to the slow but short-range translational

${ }^{\dagger}$ To whom correspondence should be addressed (E-mail: nemo@mm.kyushu-u.ac.jp). 
motion of polymeric clusters in the system.

Comparison of two sets of data below $C=30 \mathrm{wt} \%$ clearly reveals that structures of aggregates and networks are tremendously affected by valence electron number of metallic ions used for neutralization of CTPEB. Nonetheless, it remains uncertain if the slow relaxation mode is still prevailing for CTPEB-K in more concentrated region and in the bulk. We also expect that, in the highly concentrated region, ion clusters consisting of $\mathrm{K}^{+}$ions should be dominantly present, but their effects on the network structure and their relaxation process are not clarified yet.

In this work, we performed dynamic viscoelastic measurements on CTPEB-K samples with $C$ from 40 to $100 \mathrm{wt} \%$ as well as on those with $C=7$ and $10 \mathrm{wt} \%$ for establishing viscoelastic properties of this system over the whole concentration range. Extensive comparison of the data with earlier data for the CTPEB-Mg system discloses differences in the formation process of viscoelastic networks and their structures between the two ionomer systems.

\section{EXPERIMENTAL}

\section{Materials}

CTPEB-K and esterified CTPEB (CTPEB-Ester) samples were prepared from carboxyl terminated poly(ethylene butylene) copolymer (CTPEB) with the number-average molecular weight of $M_{\mathrm{n}}=1400$ $\left(M_{\mathrm{w}} / M_{\mathrm{n}} \approx 1.2\right.$, functionality $=2.15$, the ratio of ethylene to butylene units $=15: 85$, Nippon Soda Co., Ltd.,). CTPEB-Ester was prepared as ethyl carboxylate with the acylchloride method as described elsewhere. $^{21}$

CTPEB-K was prepared by complete neutralization of CTPEB adding stoichiometric amount of potassium methoxide in benzene as described elsewhere. ${ }^{21}$

Anhydrous decalin was used as solvent for preparation of CTPEB-K and CTPEB-Ester solutions with six polymer concentrations $C$ from 7 to $100 \mathrm{wt} \%$. Weighed amounts of CTPEB-K and the solvent were put into a vial and kept for at least two days so as to penetrate the solvent into CTPEB-K. Then the vial was rotated at $0.2 \mathrm{rpm}$ for about $12 \mathrm{~h}$ at $80^{\circ} \mathrm{C}$ to make samples homogeneous and transparent.

\section{Methods}

Rheological measurements were performed using a stress-controlled rheometer (Carri-MED CSL-100, UK) with a cone-and-plate type geometry of diameter $4 \mathrm{~cm}$. A solvent trap equipped with the rheometer was used to protect the sample from evaporation. The sample chamber was then covered with an external round heater box made of epoxy resin to minimize the temperature gradient in the sample.
The storage and the loss shear moduli, $G^{\prime}(\omega)$ and $G^{\prime \prime}(\omega)$, were measured in a range of angular frequency $\omega$ from 0.01 to $100 \mathrm{rad} \mathrm{s}^{-1}$ at six temperatures from $5^{\circ}$ to $55^{\circ} \mathrm{C}$. For CTPEB-K solutions with $C \geq 40 \mathrm{wt} \%$, thick films were first prepared to which $G^{\prime}(\omega)$ and $G^{\prime \prime}(\omega)$ were measured using $2 \mathrm{~cm}$ parallel plate geometry. Creep and creep recovery measurements were also conducted above $C=40 \mathrm{wt} \%$ using the same rheometer to measure viscosity.

\section{RESULTS AND DISCUSSION}

\section{Viscosity Behavior}

Solution viscosity $\eta$ of associating polymers at finite concentration can be conveniently expressed as a product of two contributions as eq 1 shows. ${ }^{22-24}$

$$
\eta=f_{1}(\zeta) \psi_{1}(C, T)
$$

Here $f_{1}(\zeta)$ is a function of the local friction factor $\zeta(C, T)$ of the segment or the single CTPEB main chain with low molecular weight and the global structure factor $\psi_{1}(C, T)$ expresses effects of attractive forces between associating polymers responsible for formation of various association structures. If the association structure formed is stable enough to keep its structure in the temperature range examined, $C$ dependence of $\psi_{1}(C, T)$ is only observed and $\psi_{1}(C, T)$ may be written as $\psi_{1}(C)$. Since the CTPEB-Ester sample has no specific intermolecular interaction at chain ends and remains as a viscous fluid in the bulk, i.e., $\psi_{1}(C, T)$ can be put equal to unity, the contribution of the structure factor to $\eta_{\text {CTPEB-M }}$ of CTPEB-M samples $(\mathrm{M}=\mathrm{K}$ or $\mathrm{Mg})$ can be extracted by taking a ratio of $\eta_{\text {CTPEB-M }}$ to $\eta_{\text {CTPEB-Ester }}$ of the CTPEB-Ester sample with the same $C$ at the same temperature. Figure 1 gives a double logarithmic plot of the ratios against $C$ for two ionomers at $55^{\circ} \mathrm{C}$. As reported earlier, ${ }^{18}$ the ratio $\eta_{\text {CTPEB-Mg }} / \eta_{\text {CTPEB-Ester }}$ increases very gradually up to $C=7 \mathrm{wt} \%$, then exhibits two steps type of viscosity enhancement, one between $C=7$ and $20 \mathrm{wt} \%$ and another between 30 and $50 \mathrm{wt} \%$, and finally becomes almost constant up to the bulk. The $\eta_{\text {CTPEB-K }} / \eta_{\text {CTPEB-Ester }}$ also gradually increases with $C$ in the low concentration region and above $C=5 \mathrm{wt} \%$, starts to increase very rapidly up to $C=40 \mathrm{wt} \%$ followed by the level-off to a value close to that of CTPEB-Mg system. We have no explanation about a small minimum at around $C=70 \mathrm{wt} \%$. The $C$ dependence of the ratio for the CTPEB-K looks to be fitted by the straight line with a slope of $4.5 \pm 0.3$ in the range of $C$ from 5 to $50 \mathrm{wt} \%$, being in good agreement with the value of $4.6 \pm 0.3 \mathrm{ob}-$ tained from a plot of $\eta_{\text {CTPEB-K }}$ against $C$ in the narrow $C$ range of $15-30 \mathrm{wt} \% .{ }^{21}$ Comparison of the two sets of data indicates that a sharp increase in $\eta$ begins to 


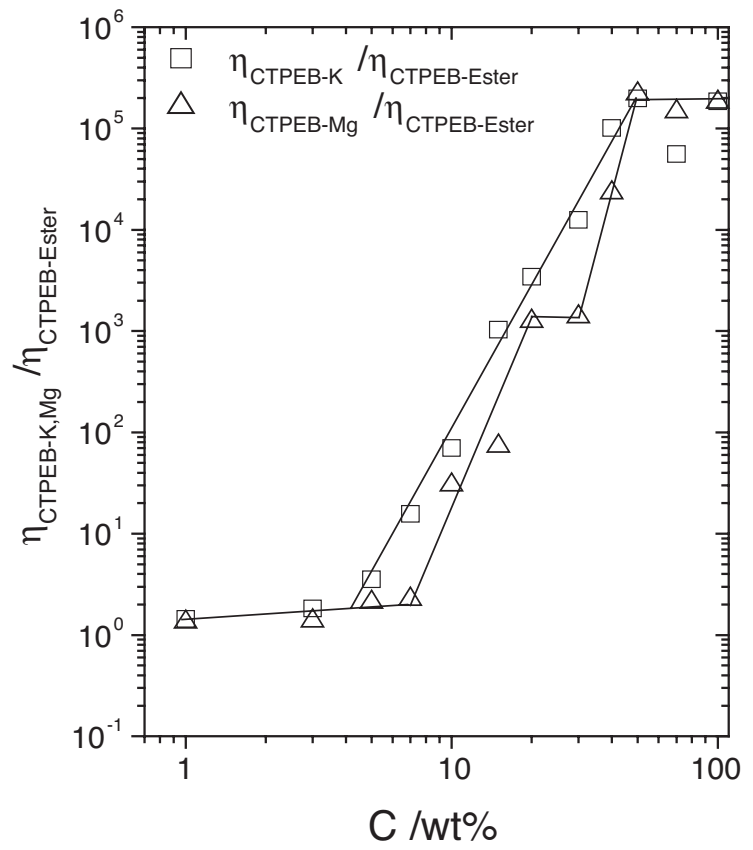

Figure 1. Concentration dependencies of viscosity ratios of CTPEB-K and CTPEB-Mg to CTPEB-Ester in decalin at $55^{\circ} \mathrm{C}$. The solid lines are empirically drawn for guidance of the eye.

occur for CTPEB-K at a lower concentration of about $4 \mathrm{wt} \%$ and values of $\eta_{\text {CTPEB-K }} / \eta_{\text {CTPEB-Ester }}$ are larger than those of $\eta_{\text {CTPEB-Mg }} / \eta_{\text {CTPEB-Ester }}$ up to $C=40$ wt $\%$. These results may be interpreted as being due to that CTPEB-K chains with monovalent $\mathrm{K}^{+}$ions can in principle form linearly associated aggregates, whereas a ring composed of five CTPEB chains is a basic unit for their linear association to the CTPEB$\mathrm{Mg}$ ionomoer. Agreement of the two ratios at higher $C$ might be fortuitous as shall be discussed later.

\section{Viscoelasticity of CTPEB-K above $C=7 w t \%$}

The angular frequency $\omega$ dependencies of $G^{\prime}$ and $G^{\prime \prime}$ of the $40 \mathrm{wt} \%$ CTPEB-K sample at temperatures $T$ from 5 to $55^{\circ} \mathrm{C}$ are shown in Figure 2. At $5{ }^{\circ} \mathrm{C}, G^{\prime}$ takes a plateau in the high frequency region, which implies that a viscoelastic network is formed over the whole volume of this solution. At an elevated temperature of $55^{\circ} \mathrm{C}, G^{\prime \prime}$ becomes larger than $G^{\prime}$ over the whole frequency range measured, indicating that the solution flows at this elevated temperature. The time-temperature superposition principle was found to be applicable for both $G^{\prime}$ and $G^{\prime \prime}$ curves of different temperatures shown in Figure 2 to obtain one composite curve by a horizontal shift alone as well as those for the solutions with $C=7-70 \mathrm{wt} \%$ in decalin and for the bulk as shown in Figure 3. The master curves of the samples with $C=7$ and $10 \mathrm{wt} \%$ show that $G^{\prime \prime}$ becomes nearly same as $G^{\prime}$ in the high frequency region, suggesting that a viscoelastic network may be formed at around $C=7 \mathrm{wt} \%$. This concentration is

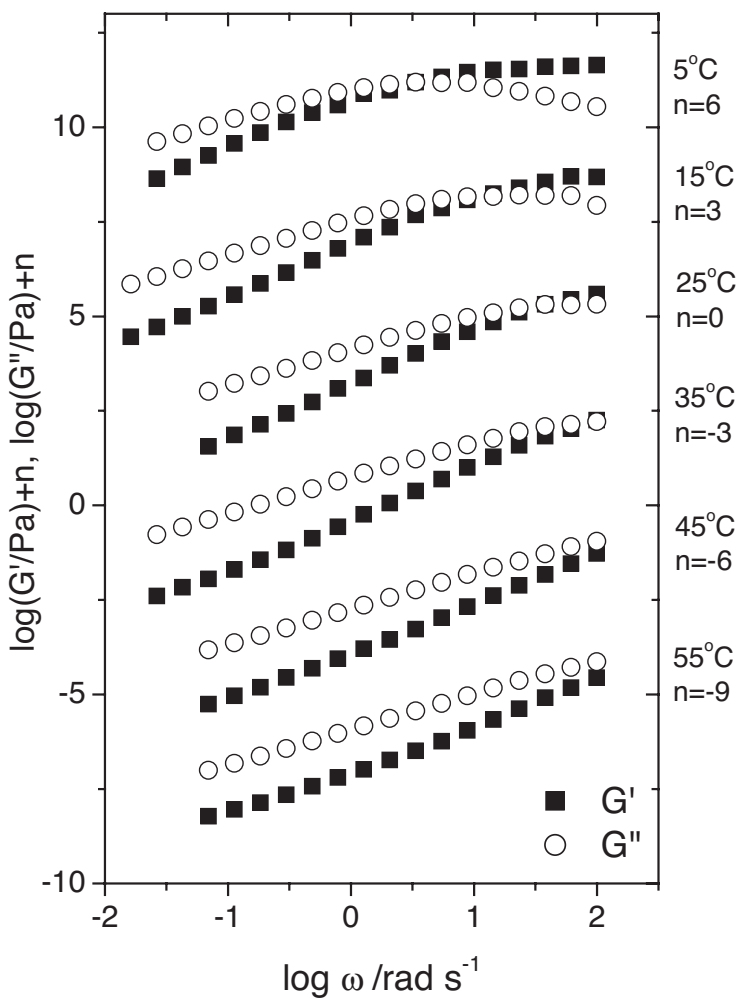

Figure 2. Angular frequency $\omega$ dependencies of $G^{\prime}$ and $G^{\prime \prime}$ for the $40 \mathrm{wt} \%$ CTPEB-K solutions in decalin at six temperatures from 5 to $55^{\circ} \mathrm{C}$. The curves are vertically shifted by $10^{n}$ for clarity of the figure.

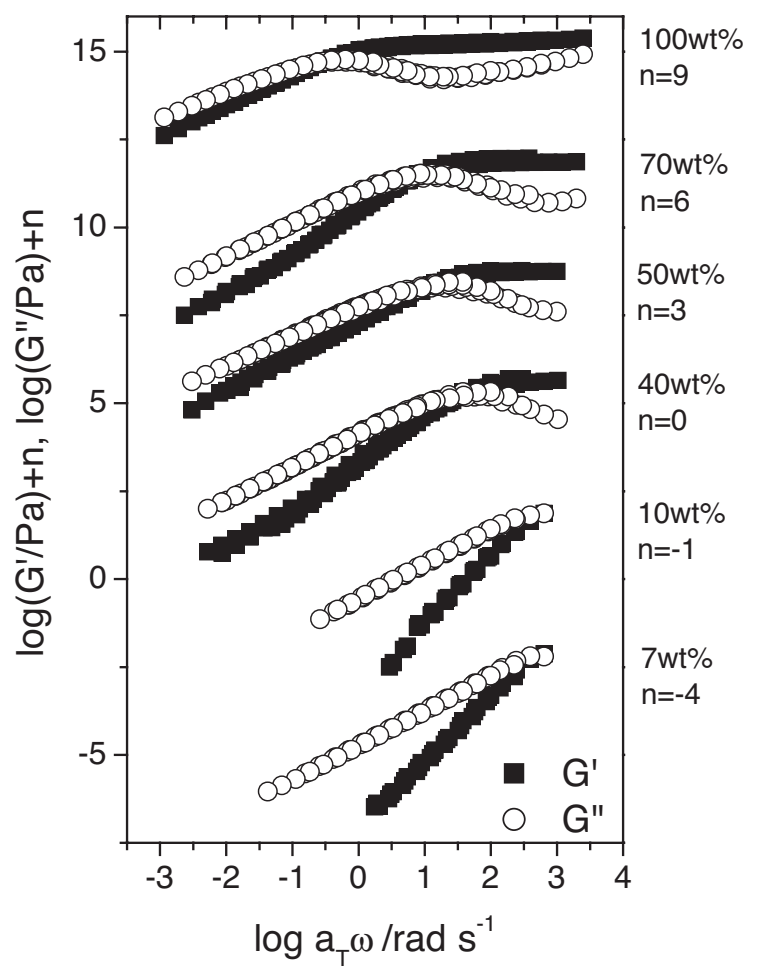

Figure 3. Master curves obtained from the time-temperature reduction scheme for CTPEB-K solutions in decalin with six concentrations from 7 to $100 \mathrm{wt} \%$. The reference temperature $T_{\mathrm{r}}$ is taken $25^{\circ} \mathrm{C}$. The curves are shifted by $10^{n}$ as indicated. 
lower than the corresponding concentration of CTPEB-Mg for which we found that the viscoelastic network is formed at around $C=15 \mathrm{wt} \%$.

The master curves of the samples with $C=40-100$ wt $\%$ reveal that a distribution of relaxation times become broader with increasing $C$ from changes in the frequency dependence of $G^{\prime}$ at low $\omega$. The dynamic viscoelastic data for CTPEB-K with $C=15-30$ wt $\%$ reported in a previous paper ${ }^{21}$ showed presence of two relaxation modes. The fast mode termed the network relaxation mode with a single relaxation time was considered to represent dissociation of a pair-wise associate of two $\mathrm{COO}^{-} \mathrm{K}^{+}$groups and the slow mode becomes observable only after the network relaxation took place. Two different models seem useful for interpretation of the relaxation mechanism of the slow mode. ${ }^{25,27}$ One is the emulsion model ${ }^{25}$ that has successfully explained viscoelasticity of the suspension system where the force between particles and the medium is repulsive. ${ }^{26}$ In this model, deformation of emulsified soft particles by applied stress is responsible for the viscoelastic response in the low $\omega$ region where $G^{\prime \prime}$ appears in proportion to $\omega$. Data fitting with this model, however, did not give a fruitful result to the CTPEB ${ }^{-} \mathrm{K}^{+}$system. On the other hand, Shikata and Pearson ${ }^{27}$ have dealt with suspensions of rigid spherical particles for which the interparticle potential is very close to the hard-core potential, and have shown that particulate random Brownian motion gives rise to viscoelasticity with a relaxation time given as $0.5 r^{2} / D$ where $D$ is the short-range translation diffusion coefficient and $r$ is the particulate radius. An analysis with this model gave about $25-30 \mathrm{~nm}$ as the average radius of polymeric clusters in the viscous solutions. Since polymeric clusters are surely deformable, the absolute magnitude may be subject to considerable uncertainty. Nevertheless, this model is not inconsistent with a picture that the network is depicted in the range of $C$ from 5 to $30 \mathrm{wt} \%$ as an assembly of polymeric clusters interconnected with linearly associated CTPEBK strands over the whole sample volume. Although there remains a trace of the slow relaxation mode in the lower frequency region of the master curve of $C=40 \mathrm{wt} \%$ sample, fitting of this Maxwell model with two relaxation modes to $G^{\prime}$ and $G^{\prime \prime}$ curves was found inapplicable for those of the solutions above $C=40 \mathrm{wt} \%$. Broad network relaxation observed in the systems above $C=40 \mathrm{wt} \%$ may be due to dissociation of larger ion clusters made of more than two $\mathrm{COO}^{-} \mathrm{K}^{+}$groups. The master curves of $C=$ 70 and $100 \mathrm{wt} \%$ samples show another relaxation mode in the high frequency region where an increase in $G^{\prime \prime}$ is seen. In spite of tremendous changes in the network structure with increasing $C$, success of the time-temperature superposition scheme strongly sug-

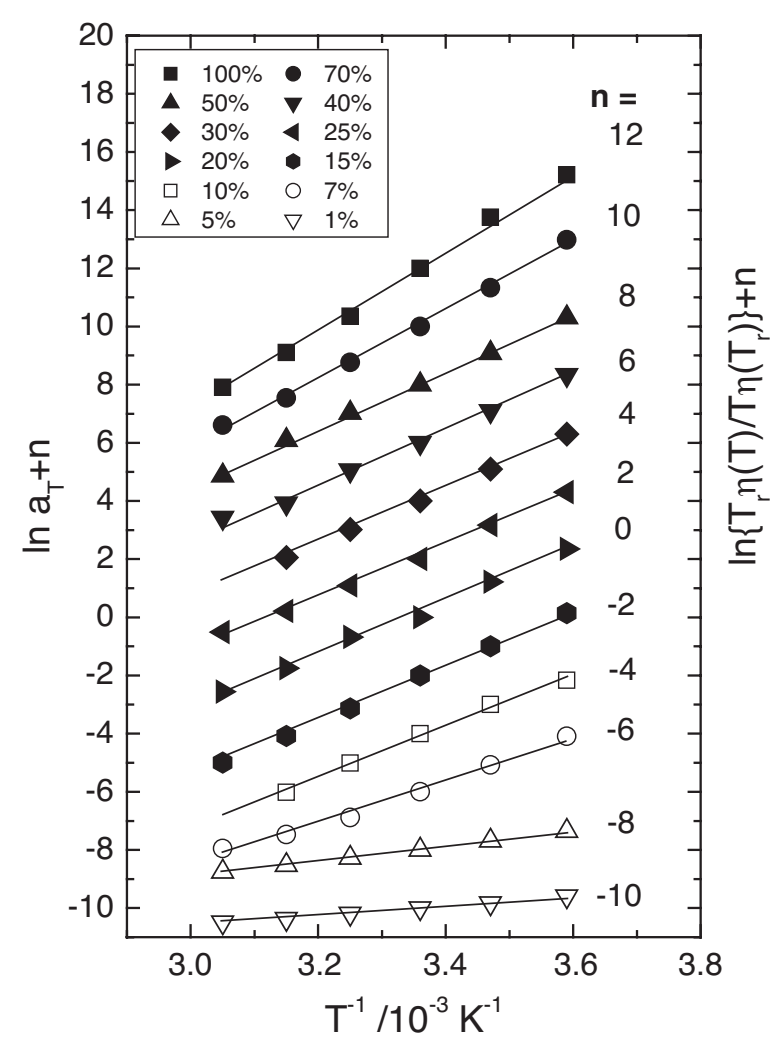

Figure 4. Semi-logarithmic plot of the shift factor, $a_{\mathrm{T}}$, against $1 / T$ for CTPEB-K solutions with $C=15-100 \mathrm{wt} \%$. Viscosity ratio $T_{\mathrm{r}} \eta(T) / T \eta\left(T_{\mathrm{r}}\right)$ is also plotted for CTPEB-K solutions with $C=$ $1-10 \mathrm{wt} \%$. The data are vertically shifted by $10^{n}$ for clarity of the figure.

gests that all relaxation modes are accompanied by the same friction mechanism, thus $\psi_{1}(C, T)$ in eq 1 is independent of $T$ in the temperature range examined and can be written as $\psi_{1}(C)$.

Figure 4 shows a semi-logarithmic plot of the horizontal shift factor $a_{\mathrm{T}}$ for $15-100 \mathrm{wt} \%$ CTPEB-K samples and the viscosity ratio $T_{\mathrm{r}} \eta(T) / T \eta\left(T_{\mathrm{r}}\right)$ for $1-$ $10 \mathrm{wt} \%$ solutions against $1 / T$ including the earlier data. ${ }^{21}$ Here, the value of $T_{\mathrm{r}} \eta(T) / T \eta\left(T_{\mathrm{r}}\right)$ should take the value same as the corresponding shift factor $a_{\mathrm{T}}$ because the time-temperature superposition principle is presumably satisfied for these samples. Figure 4 clearly shows that the temperature dependence is well described by the Arrhenius type of equation,

$$
a_{\mathrm{T}} \sim \exp \left(\frac{H}{R T}\right)
$$

where $H$ is the activation energy and $R$ is the gas constant. From slopes of the straight lines in Figure 4, we estimated $H$ for samples with different concentrations, which are listed in Table I. A semi-logarithmic plot of the viscosity ratio $T_{\mathrm{r}} \eta(T) / T \eta\left(T_{\mathrm{r}}\right)$ against $1 / T$ for CTPEB-Ester solutions with various concentrations $C$ in the range of $1-100 \mathrm{wt} \%$ were also found to follow the Arrhenius type of equation very well. Values 
Table I. Activation energy, $H$, for CTPEB-K, CTPEB-Mg, and CTPEB-Ester at different concentrations from 1 to $100 \mathrm{wt} \%^{\mathrm{a}}$

\begin{tabular}{cccc}
\hline \multirow{2}{*}{$\begin{array}{c}\text { Concentration } \\
\text { / wt } \%\end{array}$} & \multicolumn{3}{c}{$H / \mathrm{kJ} \mathrm{mol}^{-1}$} \\
\cline { 2 - 4 } & CTPEB-K & CTPEB-Mg & CTPEB-Ester \\
\hline 100 & 120 & 140 & 67 \\
70 & 98 & 113 & 38 \\
50 & 93 & 108 & 33 \\
40 & 82 & 94 & 25 \\
30 & 80 & 82 & 21 \\
20 & 75 & 76 & 18 \\
15 & 75 & 75 & 17 \\
10 & 73 & 26 & 16 \\
7 & 69 & 16 & 16 \\
5 & 22 & - & 14 \\
1 & 14 & - & 13 \\
\hline
\end{tabular}

${ }^{\mathrm{a}}$ Experimental errors lie between $\pm 0.5 \mathrm{~kJ} \mathrm{~mol}^{-1}$ for three systems.

of the activation energy calculated are also listed in Table I with corresponding values of the CTPEB$\mathrm{Mg}$ for comparison. The table shows that $H$ increases monotonously with increasing $C$ for three systems to an experimental accuracy, which gives rise to tremendous enhancement in the relaxation time $\tau$. Values of $H$ for CTPEB-K and CTPEB-Mg samples become much larger above 5 and $15 \mathrm{wt} \%$ than corresponding values of the CTPEB-Ester samples.

The $C$ dependence of $\tau$ can be discussed based on the same treatment as used for data analysis of viscosity data. The $\tau$ is conveniently expressed as the product of the local friction factor $f_{1}(\zeta)$ and the global structure factor $\psi_{2}(C, T)$. The contribution to $H$ from $f_{1}(\zeta)$ can be estimated from $H$ of the CTPEB-Ester sample. Then the contribution to $H$ from the structure factor is estimated as the activation energy difference $\Delta H$ between CTPEB-M $(\mathrm{M}=\mathrm{K}$ and $\mathrm{Mg})$ and CTPEB-Ester, which is plotted against $C$ in Figure 5. The $C$ dependence of $\Delta H$ for CTPEB-K is negligible with a constant value of $\Delta H \approx 58 \mathrm{~kJ} \mathrm{~mol}^{-1}$ from $C=7-100 \mathrm{wt} \%$. On the other hand, $\Delta H$ for CTPEB$\mathrm{Mg}$ becomes almost constant with a magnitude of $58 \mathrm{~kJ} \mathrm{~mol}^{-1}$ in a range of $C$ from 15 to $30 \mathrm{wt} \%$ followed by gradual increase up to $C=50 \mathrm{wt} \%$, and then once again levels off to an almost constant value of about $75 \mathrm{~kJ} \mathrm{~mol}^{-1}$ up to the bulk. $\Delta H$ values are found to be fairly small below $C=7$ and $15 \mathrm{wt} \%$ for CTPEB-K and CTPEB-Mg, respectively, as shown in Figure 5. This change of $\Delta H$ with $C$ may be related to a change in the relaxation mechanism of the solutions. At low concentrations before formation of viscoelastic networks, the solutions remain viscous fluid in which flow of suspension of the clusters formed by association of polymers is mainly dominated by mobility of the solvent so that the observed $\Delta H$ is fairly small. When the average size of polymeric

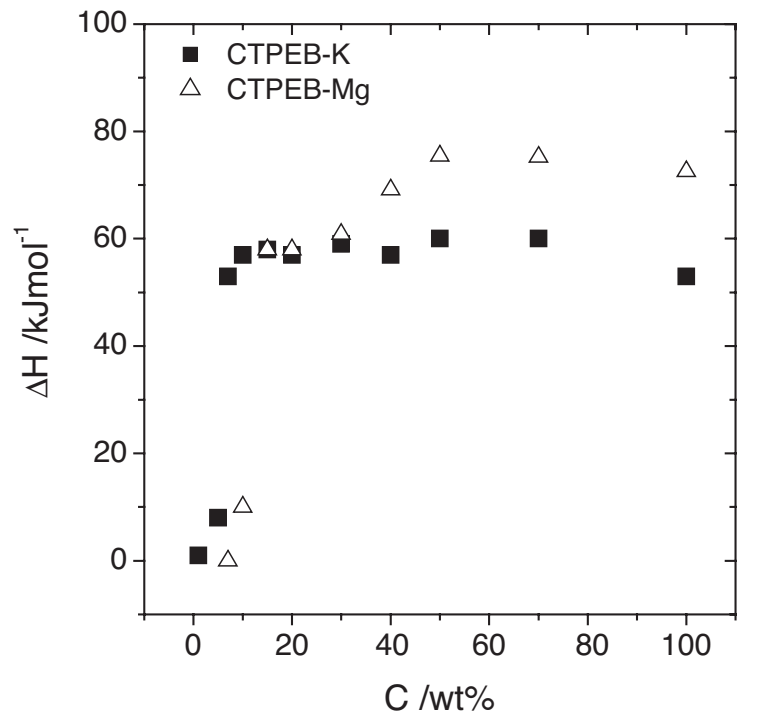

Figure 5. Concentration dependences of activation energy difference $\Delta H$ between CTPEB-K and CTPEB-Ester solutions as well as CTPEB-Mg and CTPEB-Ester solutions.

clusters increases so that an occupation ratio of space by polymeric clusters becomes close to unity, a viscoelastic network will be formed. Relaxation of network occurs via disengagement of associating points, i.e., disengagement of $\mathrm{COO}^{-} \mathrm{K}^{+}$ionic groups from ion clusters in the case of CTPEB-K system and disengagement of $\left(\mathrm{COO}^{-}\right)_{2} \mathrm{Mg}^{2+}$ groups (MDC group) from ion clusters in the case of CTPEB-Mg system. Activation energy for disengagement of associating points must be much higher than that needed for the polymeric clusters to flow in the solvent. Therefore, relaxation by disengagement of associating points gives higher $\Delta H$ values for both CTPEB-K and CTPEB-Mg samples with $C \geq 7 \mathrm{wt} \%$ and $C \geq 15$ $\mathrm{wt} \%$, respectively, in comparison with respective $\Delta H$ values at lower concentrations. As discussed in the previous paper of CTPEB-Mg, ${ }^{18}$ a weak viscoelastic network is formed at $C=15 \mathrm{wt} \%$. Relaxation of networks occurs via disengagement of two primary rings. Exchanging reaction of primary chains due to intersection of two single MDC groups belonging to different network strands may also induce relaxation. In both cases, the ionic clusters with the number of the MDC group $A=2$ is responsible for relaxation, which is supposed to correspond to lower activation energy $\Delta H=58 \mathrm{~kJ} \mathrm{~mol}^{-1}$ at $C=15-30 \mathrm{wt} \%$. The concept of linear association of primary rings becomes inapplicable when polymer chains tend to extensively overlap. Ionic clusters with $A \geq 3$ shall be formed with increasing $C$ and disengagement of the MDC bond from thermodynamically more stable clusters will need a higher activation energy $\Delta H=75$ $\mathrm{kJ} \mathrm{mol}^{-1}$ as observed above $C=50 \mathrm{wt} \%$ where primary chains must be highly overlapped in referring 
to the overlapping concentration $C^{*}=17 \mathrm{wt} \%$ for the CTPEB-Ester sample. It is to be noted that this two steps type of changes in $\Delta H$ with increasing $C$ consistently explains the anomalous viscosity enhancement of the CTPEB-Mg system shown in Figure 1. On the other hand, the CTPEB-K system showed a constant value of $\Delta H \approx 58 \mathrm{~kJ} \mathrm{~mol}^{-1}$ for $C=7-100$ wt $\%$ which is different from the $\Delta H$ value observed for the CTPEB-Mg systems above $C=30 \mathrm{wt} \%$. This strongly suggests that an escape of a $\mathrm{COO}^{-} \mathrm{K}^{+}$ionic group needs almost same $\Delta H$ value irrespective of a size of ion clusters and formation of larger ionic clusters does not stabilize $\mathrm{COO}^{-} \mathrm{K}^{+}$ionic groups appreciably in comparison with the CTPEB-Mg system.

From the high frequency plateau modulus values of $G^{\prime}$ obtained from the master curves of CTPEB-K samples, we can calculate the number density of the elastically effective chains, $v_{\text {eff }}$, using the equation $G_{\mathrm{pla}}=v_{\mathrm{eff}} k_{\mathrm{B}} T$, where $k_{\mathrm{B}}$ is the Boltzmann constant, $T$ is the absolute temperature and the front factor was assumed unity. The ratio of $v_{\text {eff }}$ to the number density of primary chains, $v$, at $25^{\circ} \mathrm{C}$ for CTPEB-K with $C$ above $15 \mathrm{wt} \%$ is shown in Figure 6. $v_{\text {eff }} / v$ appears to increase from 0.04 to 0.6 following the power law $C^{2.8 \pm 0.2}$ in a $C$ range from 15 to $40 \mathrm{wt} \%$. The values of $v_{\text {eff }} / v$ remain almost same as 0.6 for $C=40$ $70 \mathrm{wt} \%$, then once again increase up to 0.95 for the bulk. This may be related to another type of a transient crosslink as evidenced by gradual increase in $G^{\prime \prime}$ in the high $\omega$ region in Figure 3, though its dynamics is ambiguous. The behavior of $v_{\text {eff }} / v$ for CTPEB-K samples is quite different from that for CTPEB-Mg samples. As shown in the same figure, values of $v_{\text {eff }} / v$ for CTPEB-Mg are found to be as small as 0.05 at $C=$

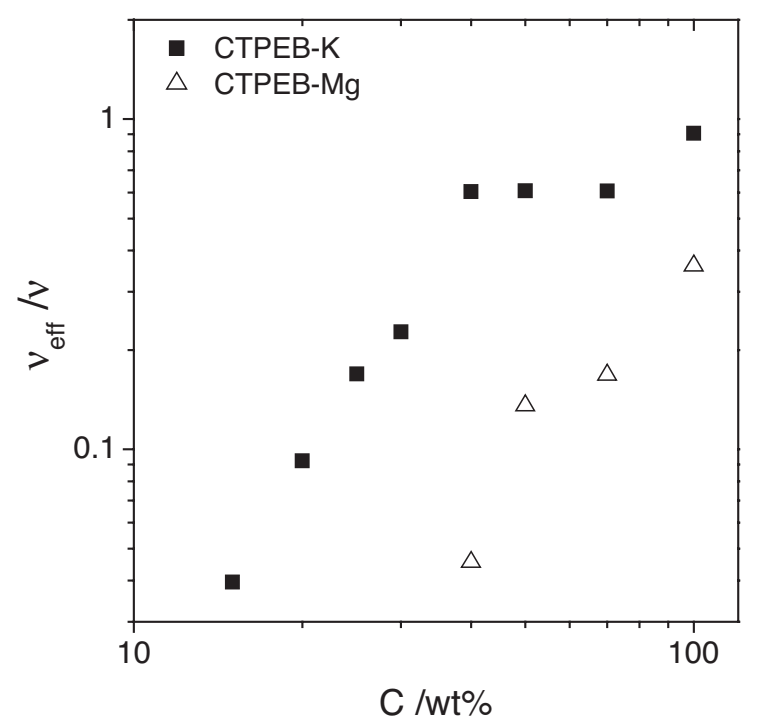

Figure 6. Concentration dependence of a ratio of the number density of elastically effective chains, $v_{\text {eff }}$, to that of primary chains, $v$, at $25^{\circ} \mathrm{C}$ for CTPEB-K and CTPEB-Mg systems.
$40 \mathrm{wt} \%$ and increased almost linearly up to 0.36 in the bulk. Thus in the bulk CTPEB-Mg sample only a fraction of chains entering into ion clusters are elastically effective, while the remaining chains may form dangling loops and are elastically ineffective.

It is well known that steady viscosity $\eta$ can be expressed as a sum of products of $i$-th relaxation time $\tau_{i}$ and an associated shear modulus $G_{i}$ in the linear viscoelastic region and $v_{\text {eff }}$ can be taken as it is in proportion to a sum of $G_{i}$ excluding contributions from the segmental relaxation modes. ${ }^{22}$ The exponents $4.5 \pm 0.3$ and $2.8 \pm 0.2$ are obtained for the power law type of the $C$ dependence of $\eta_{\text {CTPEB-K} /}$ $\eta_{\text {CTPEB-Ester }}$ in a range of $C=5-50 \mathrm{wt} \%$ and of $v_{\text {eff }} /$ $v$ in a $C$ range of $C=15-40 \mathrm{wt}$, respectively. If a distribution of relaxation times would not vary with increasing $C$, all $\tau_{i}$ s should increase approximately as $C^{1.7 \pm 0.5}$. However, this is not the case for the CTPEB$\mathrm{K}$ system to which broadening of the relaxation time distribution was observed. Indeed, curve fitting with the Maxwell model with two relaxation modes to the $G^{\prime}$ and $G^{\prime \prime}$ curves with $C$ between 15 and 30 wt $\%$ showed that $\tau_{i}$ and $G_{i}$ have very different $C$ dependencies for two modes, respectively. Evidently more elaborate theoretical analysis seems to be needed.

Viscosities of the CTPEB-K and -Mg systems are apparently close to each other at higher concentrations where ion clusters are formed. In taking into account for a large difference in $\Delta H$ between the two systems as illustrated in Figure 5, we infer that relaxation times of the CTPEB-Mg system may be much longer than that of the CTPEB-K system. On the other hand, the reverse is true for $v_{\text {eff }} / v$. It is likely that the product of two quantities might have taken the values accidentally close to each other.

Acknowledgment. The authors are grateful to Nippon Soda Co., Ltd., Tokyo, Japan for a gift of CTPEB samples.

\section{REFERENCES}

1. A. Eisenberg and J.-S. Kim, "Introduction to Ionomers," Wiley Interscience, New York, N.Y., 1998.

2. M. R. Tant, K. A. Mauritz, and G. L. Wilkes, "Ionomers: Synthesis, Structure, Properties and Applications," Blackie Academic \& Professional, New York, N.Y., 1997.

3. S. Schlick, "Ionomers: Characterization, Theory, and Application," CRC Press Inc., Boca Raton, FL, 1996.

4. A. Eisenberg and M. King, in "Ion-Containing Polymers, Polymer Physics, Vol. 2,” R. S. Stein, Ed., Academic Press, New York, N.Y., 1977.

5. M. Hara, J. Wu, Y. Wang, R. Jerome, and M. Granville, Polym. Prepr. (Am. Chem. Soc., Div. Polym. Chem.), 30, 219 (1989). 
6. G. Broze, R. Jerome, and P. Teyssie, Macromolecules, 14, 224 (1981).

7. G. Broze, R. Jerome, and P. Teyssie, Macromolecules, 15, 920 (1982).

8. M. Moller, E. Muhleissen, and J. Omeis, in "Physical Networks Polymers and Gels," W. Burchard and S. B. RossMurphy, Ed., Elsevier Applied Science, London, U.K., 1990, pp 45-64.

9. M. R. Tant, G. L. Wilkes, R. Storey, and J. P. Kennedy, Polym. Bull., 13, 541 (1985).

10. R. D. Hedegus and R. W. Lenz, J. Polym. Sci., Polym. Chem. Ed., 26, 367 (1988).

11. E. Karayianni, R. Jerome, and S. L. Cooper, Macromolecules, 28, 6494 (1995).

12. E. Karayianni, R. Jerome, and S. L. Cooper, Macromolecules, 30, 7444 (1997).

13. E. Karayianni, R. Jerome, and S. L. Cooper, Macromolecules, 33, 6473 (2000).

14. G. Broze, R. Jerome, P. Teyssie, and C. Mrco, Macromolecules, 16, 996 (1983).

15. M. R. Tant, G. L. Wilkes, M. D. Read, and J. P. Kennedy, J. Polym. Sci., Polym. Lett. Ed., 24, 19 (1986).
16. M. R. Tant, G. L. Wilkes, and J. P. Kennedy, J. Appl. Polym. Sci., 42, 523 (1991).

17. S. A. Rahman, A. Takada, and N. Nemoto, J. Soc. Rheol., Jpn., 30, 95 (2002).

18. S. A. Rahman, H. Kitao, and N. Nemoto, Polymer, 45, 4523 (2004).

19. H. Yamakawa, "Modern Theory of Polymer Solutions," Harper \& Row, New York, N.Y., 1971.

20. H. Fujita, "Polymer Solutions," Elsevier, Amsterdam, 1990.

21. S. A. Rahman and N. Nemoto, J. Soc. Rheol., Jpn., 31, 219 (2003).

22. J. D. Ferry, "Viscoelastic Properties of Polymers 3rd ed.," John Wiley \& Sons, New York, N.Y., 1980.

23. G. C. Berry and T. G. Fox, Adv. Polym. Sci., 5, 261 (1968).

24. N. Nemoto, T. Kojima, T. Inoue, and M. Kurata, Polym. J., 20, 875 (1988).

25. D. Grambling, R. Muller, and J. F. Palierne, Macromolecules, 26, 320 (1993).

26. T. Matsumoto, "Rheology of Dispersion Systems," Kobunshi Kankokai, Kyoto, 1997 (in Japanese), chapt. 4.

27. T. Shikata and D. S. Pearson, J. Rheol., 38, 601 (1994). 